\title{
Mistaking Reversed Halo Sign with Cavity in Organizing Pneumonia: A Case Report
}

\author{
Mohammad Badr Almoshantaf ${ }^{a}$ Mukhtar Almosli ${ }^{\mathrm{b}}$ Ghanem Aljamali ${ }^{\mathrm{b}}$ \\ ${ }^{a}$ Department Emergency, Syrian Arab Red Crescent, Homs, Syria; ${ }^{b}$ Alkendi Hospital, Homs, Syria
}

\section{Keywords}

Organizing · Pneumonia · Organizing pneumonia ·

Reversed halo sign · Cavity

\begin{abstract}
Organizing pneumonia (OP) is a rare inflammatory lung condition affecting bronchioles and alveoli. Symptoms may include cough, fever, malaise, fatigue, and weight loss with a wide variety of radiological manifestations. Both the unspecific symptoms and the radiological manifestations wide spectrum can make the diagnosis of OP challenging in daily practice. A pathological biopsy is a gold standard for diagnosis. However, there is usually a delay in making the diagnosis as a result of the time that is taken to decide to perform the biopsy. We present a case of OP in which its radiological manifestation first was mistaken for a cavity instead of the reversed halo sign (atoll sign) due to the similarity in some radiological features.

(C) 2021 The Author(s).

Published by S. Karger AG, Basel
\end{abstract}

\section{Introduction}

Organizing pneumonia (OP) is a reaction to lung injury in which the alveolar epithelium produces intraluminal plugs of granulation tissue (also called Masson bod- ies). This granulation progresses to reach the lumen of bronchioles, alveolar ducts, and adjacent peribronchiolar alveoli, causing an inflammatory effect [1]. OP can be generally classified into 2 types: cryptogenic organizing pneumonia $(\mathrm{COP})$ and secondary organizing pneumonia (SOP). COP is the type of OP when the cause of injury is unknown, meaning that $\mathrm{COP}$ diagnosis can only be made after the exclusion of any possible etiology. While SOP is diagnosed when a specific cause is identified such as infections, toxic gases, drugs, radiation, etc. [2]. Presenting symptoms usually are fever, fatigue, nonproductive cough, mild dyspnea, and weight loss. OP affects equally both genders, typically in their sixth decade of life, with a subacute course duration of $<3$ months [3]. The radiologic manifestation of OP varies. The most common of which are consolidations and ground-glass opacities. Other appearances like perilobular opacities, reversed halo (atoll) sign, nodular opacities, parenchymal bands, bronchial walls thickening, and masses have also been observed in some OP cases [2]. Because of the clinical similarity that OP shows to bacterial pneumonia, in addition to the wide radiological variation, the diagnosis of $\mathrm{OP}$ becomes more challenging and needs the combination of clinical features, imaging, and pathology [3,4]. Generally, corticosteroids are the standard treatment. But may induce relapse in more than half of the cases. On the other hand, clarithromycin (macrolides) has been reported to karger@karger.com www.karger.com/dmj

Karger $\stackrel{\text { ' }}{5}$

GOPEN ACCESS
(C) 2021 The Author(s)

Published by S. Karger AG, Basel

This article is licensed under the Creative Commons AttributionNonCommercial-NoDerivatives 4.0 International License (CC BYNC-ND) (http://www.karger.com/Services/OpenAccessLicense) Usage and distribution for commercial purposes as well as any distribution of modified material requires written permission.
Mohammad Badr Almoshantaf

Department Emergency, Syrian Arab Red Crescent 2nd islet Muhamad Al-Ashmar St

Al Waer, Homs (Syria)

baderalmoushantaf1995@gmail.com 
have fewer relapse cases with better-tolerated therapy. However, clarithromycin showed ineffectiveness in some patients [5]. Other immunosuppressive therapies, such as cyclophosphamide and cyclosporine, also seem to be effective [6].

We present a case of OP that at the first glance appeared as an isolated cavity-like lesion in chest X-ray and differential diagnosis has been considered accordingly. Even after the CT was done, the reversed halo sign has not yet been recognized as it was relatively difficult to distinguish from a cavity. OP has only been diagnosed after a transbronchial biopsy which made us review the imaging.

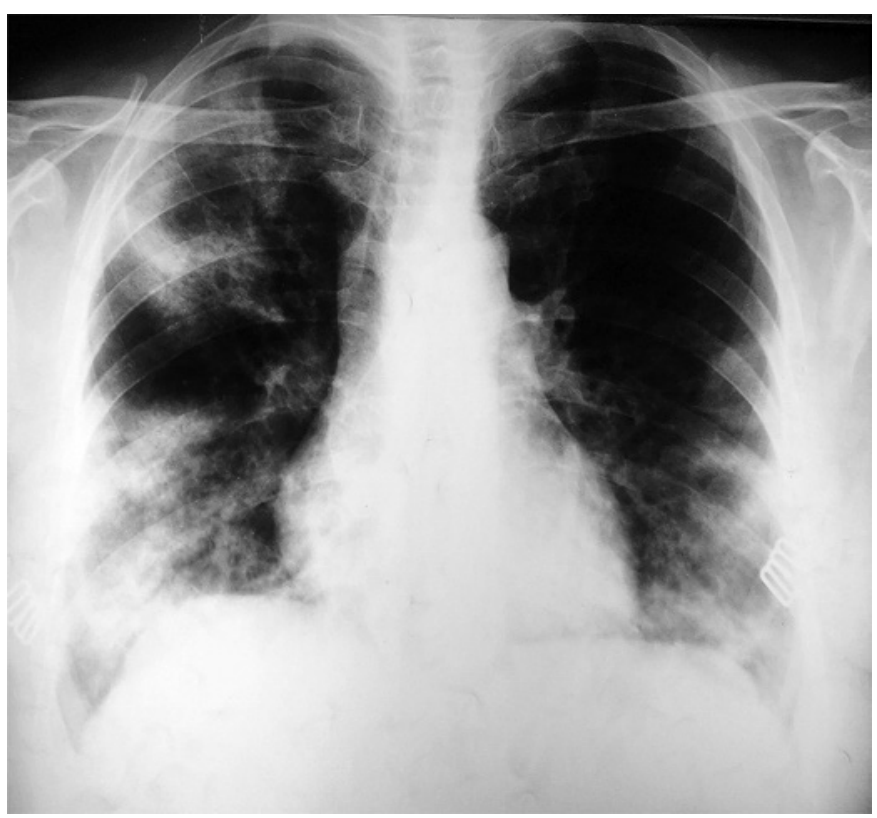

Fig. 1. Chest X-ray on admission showing bilateral basal reticulonodular infiltrations and a cavity-like opacity in the right upper lobe.

\section{Case Report}

The authors present a case of a 56-year-old smoker female with no history of pulmonary diseases. She visited the outpatient clinic with symptoms of cough, coryza, generalized weakness, and continued somnolence for the last 20 days. She has also stated that she unintentionally lost $10 \mathrm{~kg}$ of weight during that period. On physical examination, fine crackles have been heard when examining respiratory system. Rest oxygen saturation was $94 \%$. She had an overweight BMI. Examination of other systems was normal.

Investigations revealed WBCs of $13,000 / \mathrm{mm}^{3}$ with neutrophils dominance, ESR: $90 \mathrm{~mm}$ at the end of $1 \mathrm{~h}$, CRP: $25.7 \mathrm{mg} / \mathrm{dL}$, and slightly elevated LDL and triglycerides. Other investigations such as glucose, urea, $\mathrm{Cr}$, uric acid, electrolytes, ALT, AST, hemoglobin, and hematocrit, all were within normal limits. Chest X-ray showed bilateral basal reticulonodular infiltrations and a cavity-like opacity in the right upper lobe which suggested TB. No effusions or lymphadenopathy had been observed (Fig. 1).

A contrast-enhanced chest CT has been performed and revealed basal bilateral unenhanced $10-12 \mathrm{~mm}$ uncalcified nodules that were mostly peripheral with alveolar consolidations around them showing multifocal reversed halo signs (Fig. 2), in addition to 1 isolated $60-\mathrm{mm}$ well-enhanced thick-walled lesion in the upper right lobe, which was irregular from the outside and had an empty smooth inner center with no fluid level; this lesion has later been recognized as reversed halo sign (Fig. 3).

Before recognizing the reversed halo sign, no clear radio-clinical link and no definite diagnosis has been made as the main lesion was being seen as a cavity. Differential diagnoses were infectious diseases (bacterial pneumonia, active tuberculosis, and fungal infection), neoplasms, and immunological diseases (granulomatosis polyangiitis). After further tests, tuberculin skin test came back negative. P-ANCA and C-ANCA were also negative. Abdominal echography was normal. So, bronchoscopy has been planned for the following week and the pathological results of the bronchial aspirate and transbronchial biopsy concluded OP as a diagnosis after the following findings:

- Macroscopy: non-turbid and non-bloody mucinous material.

- Microscopy: no neoplastic lesions; advanced bronchial alveolar inflammatory process has been observed and manifested by the following: abundant background of cellular inflammatory infiltrate and fibroblastic plugs in alveolar sacs and ducts com-
Fig. 2. CT scan after admission showing basal bilateral unenhanced 10-12-mm uncalcified nodules with alveolar consolidations around them showing multifocal reversed halo signs.
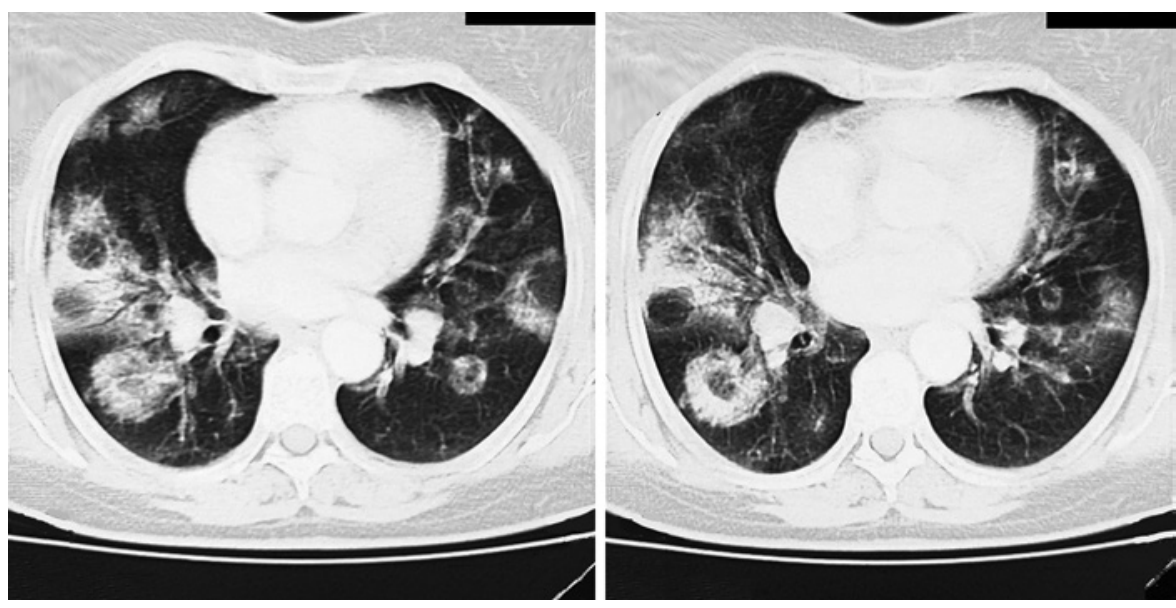

Mistaking Reversed Halo Sign with Cavity in Organizing Pneumonia
Dubai Med J 2021;4:64-67

DOI: $10.1159 / 000513428$ 

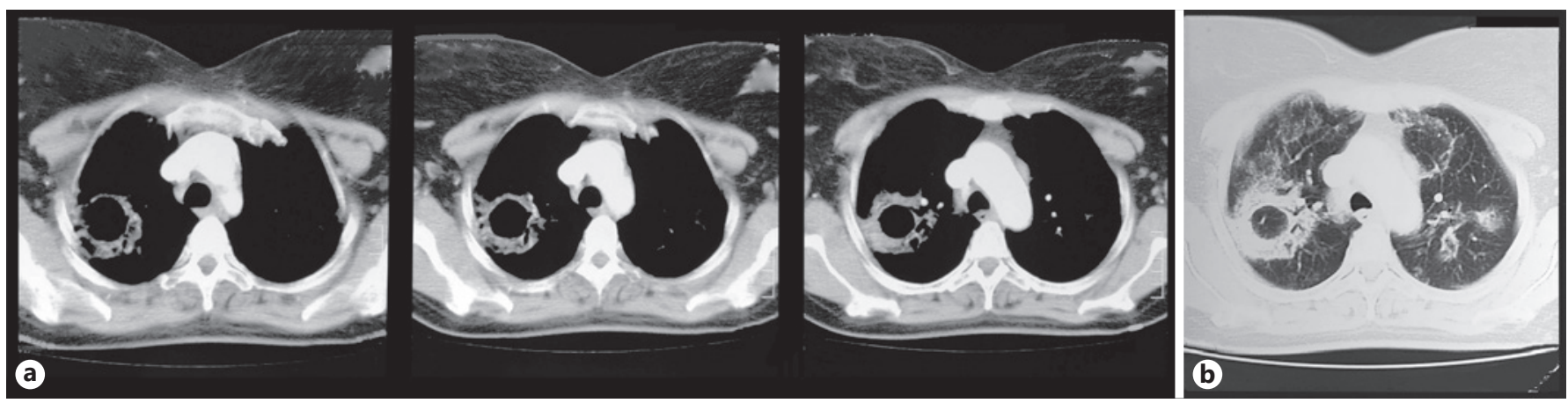

Fig. 3. a CT scan after admission showing 1 isolated $60-\mathrm{mm}$ well-enhanced thick-walled lesion in the upper right lobe, which was irregular from the outside and had an empty smooth inner center with no fluid level showing reversed halo sign. b Same CT scan with lung window showing interstitial parenchyma within the lesion.

prise spindled fibroblasts in pale matrix of loose collagen in elongated forms.

After the biopsy, we deeply reviewed the CT imaging and concluded that our cavitary lesion was in fact a typical reversed halo sign which is in keeping with OP diagnosis. Thus, a 2-month prednisolone course has been initiated as follows: $30 \mathrm{mg}$ daily for 30 days, $20 \mathrm{mg}$ daily for 20 days, and $10 \mathrm{mg}$ daily for 10 days. Additionally, a 3-day course of Azithromycin $500 \mathrm{mg}$ has been also applied.

In her first follow-up appointment, after 1 month under medication, results of investigations came with ESR: 40 at the end of $1 \mathrm{~h}$ and CRP: $12.4 \mathrm{H}$ indicating improvement. Compatibly, chest $\mathrm{X}$-ray showed resolution of the previously observed bilateral basal opacities and right epical lesion with remnants of some interstitial basal opacities (Fig. 4).

In her second follow-up appointment, after 1 month of finishing her medication course, she reported symptoms relief and weight regain. Both chest X-ray and chest CT were normal with some remnants (Fig. 5). She maintains regular evaluation with pneumology appointments and presently has no respiratory or other relevant complains.

\section{Discussion}

The OP has several radiological manifestations. In our case, OP was manifested by reversed halo sign. When observing the chest X-ray imaging, we noticed a typical cavity sign (Fig. 1) and mediastinal window of the CT imaging went along with it (Fig. 3a). However, after the result of the biopsy, we deeply reviewed the imaging and 1 slice of lung window imaging slightly showed interstitial parenchyma within the lesion (Fig. 3b), confirming reversed halo sign. We believe that the absence of high-resolution CT imaging may was a factor in confusing radiologic features. Transbronchial biopsies were recommended since the finding of characteristic intra-alveolar buds at histo-

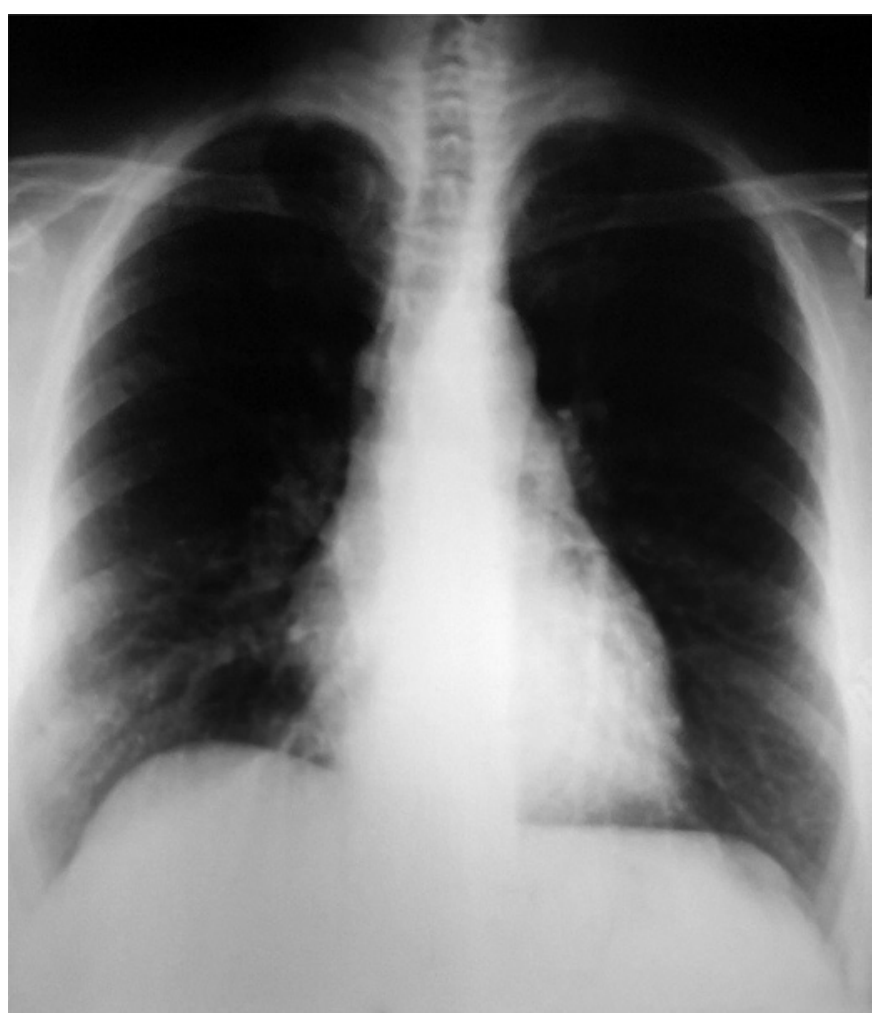

Fig. 4. Chest X-ray after treatment showing resolution of the previously observed bilateral basal opacities and right epical lesion with remnants of some interstitial basal opacities.

pathological examination is sufficient in most cases to make a provisional diagnosis of OP $[7,8]$. However, it does not usually allow exclusion of other histopathological processes coexisting with foci of OP [6]. Therefore, that we were unable to say for certain if it was COP or 
Fig. 5. CT scan after treatment showing resolution of both basal and epical manifestations.
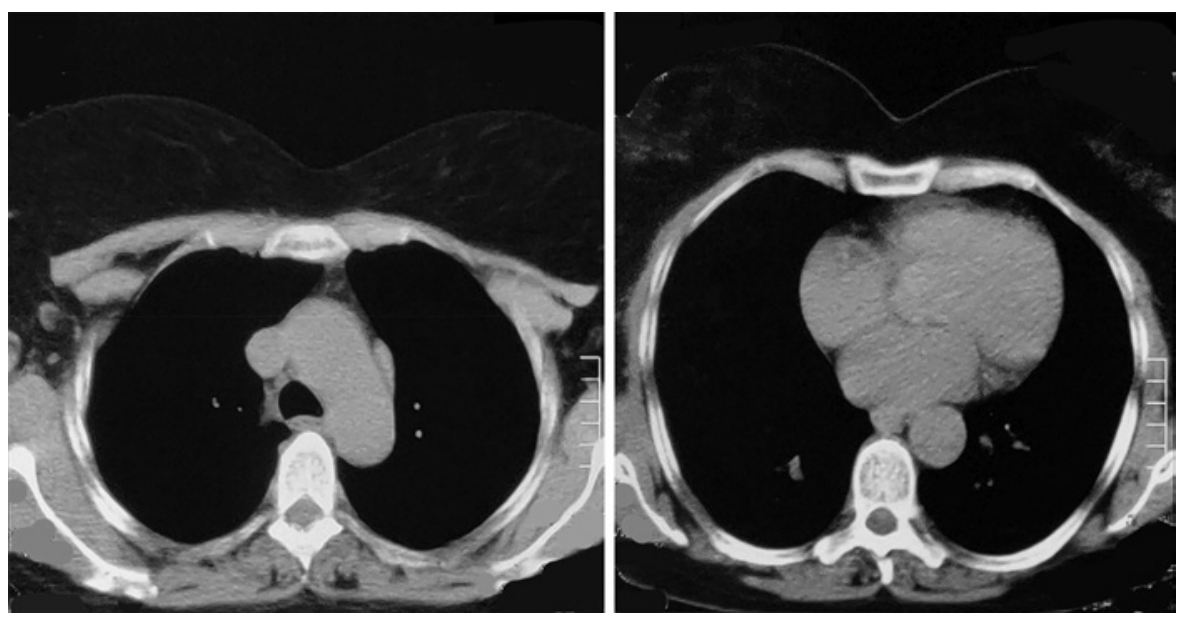

SOP. Corticosteroid therapy led to a remarkable clinical and radiologic resolution. We do not know how Azithromycin alone would have affected the outcome as it was applied through corticosteroid therapy. No relapse has been reported to the moment. The complete resolution with corticosteroids plus no relapsing of symptoms both make the diagnosis of COP more probable than SOP.

\section{Conclusion}

This case demonstrates that the wide variety of $\mathrm{OP}$ radiological manifestations may require a better diagnosis protocol and equipment to detect and clarify OP as early as possible.

\section{Acknowledgements}

The authors would like to thank Dr G.A., anesthesiologist, Homs, Syria, and Dr A. A. intensive care specialist, Homs, Syria, for their wonderful support.

\section{Statement of Ethics}

Taken from Hospital Ethics Committee. The patient's written consent was taken for publication.

\section{Conflict of Interest Statement}

None declared.

\section{Funding Sources}

The authors did not receive any funding.

\section{Author Contributions}

M.B.A. took the lead in writing the manuscript and collecting the relevant data and references. M.A. carried out the biopsy cultivate procedure and treatment and reviewed and edited the pathological part of the case. G.A. carried out the chest X-ray and the $\mathrm{CT}$ imaging, provided imaging reports, and reviewed the radiological part of the case.

\section{References}

1 Izykowski N, Kuehnel M, Hussein K, Mitschke K, Gunn M, Janciauskiene S, et al. Organizing pneumonia in mice and men. J Transl Med. 2016;14(1):169-9.

2 Zare Mehrjardi M, Kahkouee S, Pourabdollah M. Radio-pathological correlation of organizing pneumonia (OP): a pictorial review. $\mathrm{Br} \mathrm{J}$ Radiol. 2017;90(1071):20160723-3.

3 Ailing L, Ning X, Tao Q, Aijun L. Successful treatment of suspected organizing pneumonia in a patient without typical imaging and pathological characteristic: a case report. Respir Med Case Rep. 2017;22:246-50.
4 Kang C, Vali Y, Naeem M, Reddy R. Cryptogenic organising pneumonia presenting with spontaneous pneumothorax and the value of procalcitonin: a case report. Respir Med Case Rep. 2017;22:36-8.

5 Radzikowska E, Wiatr E, Langfort R, Bestry I, Skoczylas A, Szczepulska-Wójcik E, et al. Cryptogenic organizing pneumonia-Results of treatment with clarithromycin versus corticosteroids-Observational study. PLoS One. 2017;12(9):e0184739-e39.
6 Cordier JF. Cryptogenic organising pneumonia. Eur Respir J. 2006;28(2):422-46.

7 Dina R, Sheppard MN. The histological diagnosis of clinically documented cases of cryptogenic organizing pneumonia: diagnostic features in transbronchial biopsies. Histopathology. 1993;23(6):541-5.

8 Azzam ZS, Bentur L, Rubin AH, Ben-Izhak O, Alroy G. Bronchiolitis obliterans organizing pneumonia. Diagnosis by transbronchial biopsy. Chest. 1993;104(6):1899-901. 